\title{
How well do we know reaction pathways in metamorphic rocks?
}

\section{MATTHIAS KONRAD-SCHMOLKE ${ }^{1}$}

${ }^{1}$ Department of Earth Sciences, University of Gothenburg, mks@gvc.gu.se

There is still an ongoing debate about characterization and quantification of reaction rate controlling parameters in metamorphic rocks. Recent developments in analytical techniques allow for the observation of element exchange during mineral reactions down to the nano-scale, which led to the discovery of entirely new reaction pathways among solids and fluids. I will present examples from natural rocks, which clearly indicate that: (1) reaction induced lattice defects, such as dislocation walls in the reacting mineral allow for very fast intracrystalline element transport and very high dissolution rates of the reactant, (2) transient and interconnected metamorphic porosity enables effective element transport during rock transformation, (3) the formation of an amorphous transport medium during mineral reactions, that is thermodynamically decoupled from an inter-crystalline aqueous solution, leads to enhanced element transfer between reactant and product, and (4) re-

polymerization of that amorphous phase at the product surface further enhances the crystallization rate during metamorphic reactions.

All of these observations indicate that metamorphic mineral reactions might follow different pathways with different reaction rates, which might be much more effective and faster than previously thought. It is noteworthy that subduction related low temperature/high pressure rocks are most valuable sources of information about reaction pathways as peak temperatures of these rocks are sufficiently low to allow for the preservation of reaction textures, whereas high fluid fluxes and peak pressures allow for large chemical potential differences hence still invoking mineral reactions. 
This abstract is too long to be accepted for publication.

Please revise it so that it fits into the column on one page. 